\title{
LA INVESTIGACIÓN SOBRE FORMACIÓN DOCENTE EN CHILE. TERRITORIOS EXPLORADOS E INEXPLORADOS*
}

\section{Tatiana Cisternas ${ }^{1}$}

\begin{abstract}
RESUMEN
Pese al impulso que ha tenido en los últimos años la discusión en torno a la preparación de profesores, la investigación que tiene como propósito estudiar la formación inicial y continua de ellos se presenta como una dimensión escasamente explorada en el escenario nacional. ¿Qué características tiene la investigación en este campo? El presente artículo sintetiza parte de los hallazgos de un trabajo de investigación doctoral cuyo propósito es establecer los rasgos distintivos de los objetos que esta problematiza y estudia. A partir de 82 resúmenes de investigaciones realizadas entre los años 1996 y 2007, el conjunto de los estudios sobre formación docente en Chile muestra algunos focos más explorados que otros y una tendencia a construir problemas unidimensionales, es decir, donde la aproximación al fenómeno se realiza observando solo uno de los componentes involucrados en la formación.
\end{abstract}

Palabras clave: investigación, formación docente, objetos de estudio, meta-análisis

\section{RESEARCH ON TEACHERS' EDUCATION IN CHILE: KNOWN AND UNKNOWN TERRITORIES}

\begin{abstract}
Despite the strength shown by the debate about teacher preparation in recent years, research aimed to study the initial and continuing training of teachers is presented as a poorly explored dimension in the national scenario. Which are the research characteristics in this field? This article synthesizes some of the findings of a doctoral research work aimed at setting the features of objects that it problematizes and studies. From 82 summaries of research made between 1996 and 2007, it seems that all the studies of teacher education in Chile explore some items more than others and tend to build one-dimensional problems, that is to say they approach the phenomenon of formation only from one perspective of components.
\end{abstract}

Keyword: research, teacher training, study objects, meta analysis

* Estudio financiado por Conicyt, mediante la Beca de Formación de Posgrado: Investigación sobre formación docente en Chile. Una aproximación a partir de la construcción de sus objetos de estudio.

1 CIDE / Facultad de Educación. Universidad Alberto Hurtado. Santiago, Chile.

Contacto: tatiscister@gmail.com 


\section{LA INVESTIGACIÓN SOBRE FORMACIÓN DOCENTE EN CHILE. TERRITORIOS EXPLORADOS E INEXPLORADOS}

\section{Introducción}

A continuación se presentan los hallazgos de un trabajo de investigación doctoral cuyo propósito era comprender los procesos de investigación en el campo de la formación docente a partir de las características presentes en los objetos que han sido problematizados en ese campo durante los últimos años en nuestro país y desde las condiciones que subyacen a la definición de estos particulares objetos de investigación. Consideramos que una manera de comprender el desarrollo de este campo de estudio es reconociendo la diversidad de problemas y objetos que son estudiados. Específicamente, en este artículo presentamos una parte de nuestros resultados, los que muestran las direcciones que ha seguido la investigación sobre formación docente realizada en Chile en el periodo 1996-2007.

En un primer momento, identificaremos las tensiones que justifican nuestra pregunta de investigación para luego enmarcar nuestro objeto en las discusiones nacionales e internacionales. Destacaremos igualmente la singularidad y juventud de la formación docente, como campo de estudio. Con posterioridad indicaremos las preguntas y propósitos que guiaron este estudio, junto con las decisiones metodológicas implicadas. Como nuestro objetivo es presentar los hallazgos más relevantes de este estudio, haremos una descripción general del corpus de investigaciones analizadas, sugiriendo tendencias y variables sobre el desarrollo del campo en nuestro país. Para ello responderemos a la pregunta ¿qué objetos han sido problematizados por la investigación sobre formación docente en nuestro país en los últimos años? Basados en esta interrogante revelaremos cómo se manifiestan dichos focos de preocupación mediante los actores que participan en la formación, los dispositivos 
o mecanismos y los saberes profesionales que constituyen este fenómeno. En los dos últimos apartados señalaremos nuestras principales conclusiones llamando la atención sobre aquello que "no se deja ver" en los estudios a la formación docente, sugiriendo preguntas que puedan aportar a la agenda investigativa en este campo.

\section{¿Por qué analizar las investigaciones sobre formación docente en Chile?}

Adoptar una posición interrogativa y analítica respecto de la investigación en este campo, supone entender la tríada políticasprácticas-investigación como un modelo para comprender, intervenir, transformar y construir conocimiento en el ámbito de la formación de profesores. Si bien pocos cuestionan la relevancia tanto de las prácticas de formación como de las políticas que regulan a las mismas, lo cierto es que la investigación que tiene por objeto la formación docente, se presenta como una dimensión nueva, poco reconocida y muchas veces desatendida. Pese a ello, durante los últimos años han aflorado diversas perspectivas teóricas y empíricas en torno a esta arista, ubicándola en espacios estratégicos dentro de los debates sobre la formación y el desarrollo profesional docente.

En efecto, junto a las políticas y prácticas desarrolladas en este campo, diversos autores señalan que la investigación que tiene por objetivo la formación de los profesores, paulatinamente se ha ido configurando como un eje central para el desarrollo de esta área (Ávalos, 1997; Cochran-Smith, 2001, 2002; Cochran-Smith y Demers, 2008; Cochran-Smith y Fries, 2005; Huberman, 1987; Lenoir y Vanhulle, 2005; Tardif, 2004; Zeichner, 1999). Más aún, adherimos a quienes afirman que se trata de una de las discusiones más importantes y urgentes en torno a la formación de profesores, por cuanto plantea desafíos y debates para la construcción de una base de investigación que informe, ilumine, oriente y/o justifique determinadas políticas y prácticas de formación, aportando con respuestas contextualizadas y pertinentes a las particulares realidades de los sistemas educativos (Borko, Whitcomb y Kathryn, 2008; Cochran-Smith, 2002). 
El protagonismo de esta dimensión (el estudio sistemático de la formación) se ha traducido en dos fenómenos interrelacionados: por una parte, observamos el incremento de investigaciones que se ocupan de este objeto, y por otra, el creciente interés por revisar, sistematizar y comprender cuáles son los recorridos y opciones que caracterizan y distinguen esta particular área de estudio (Zeichner, 1999). En nuestro país, encontramos señales contradictorias en relación con estos dos fenómenos. Observamos un aumento sostenido de investigaciones que interrogan la formación de profesores, y a la vez escasean las iniciativas individuales y colectivas de revisión, reflexión o crítica a las prácticas y los productos de la investigación sobre formación docente en particular y de la investigación educativa en general.

La atención cada vez más frecuente hacia la formación docente como área de estudio se produce en un contexto de crítica y demanda respecto a la renovación de los procesos de formación de profesores. Se sostiene, por ejemplo, que la preparación de profesores articula débilmente la formación pedagógica y la formación en la especialidad; que el nivel de aprendizaje de los contenidos curriculares y la comprensión de la naturaleza teóricopráctica de la acción pedagógica resultan insuficientes; que las relaciones con el sistema educativo son aún incipientes y que pese a las mediciones externas (como la prueba INICIA), no se ha avanzado en procedimientos internos confiables de monitoreo y evaluación a los programas de formación. Se argumenta que, al menos en parte, estas debilidades se deberían a la escasa actualización de los programas de formación y a una débil capacidad instalada tanto para producir conocimiento desde las propias escuelas de pedagogía, como para asimilar e incorporar a la formación el aporte de investigaciones sobre aprendizaje, enseñanza, la escuela y el trabajo docente (Comisión Formación Docente, 2005; Lara, Förster y Gorichon, 2007; Mineduc, 2006; OCDE, 2004). Asimismo, en la última década la intensificación de los debates y cuestionamientos a la calidad de los procesos ha venido de la mano con un aumento sostenido de estudios que atienden a los procesos de enseñanza $y$, en particular, a los referidos al profesor y a su formación (CIDE y Mineduc, 2007). Esto se ha visto reforzado con un incremento 
en los incentivos para la constitución de centros de investigación especializados en el ámbito educativo, los cuales han desarrollado agendas específicas en torno al desarrollo profesional docente y su formación ${ }^{2}$.

Si bien, para algunos se trata de un aumento poco significativo que refleja la precariedad y el incipiente desarrollo de la investigación educativa en nuestro país, para otros es una señal esperanzadora que evidencia el impulso que ha tenido esta área de estudio. Sea cual sea la opinión del lector, se refleja una tendencia al incremento de investigaciones que abordan la formación docente. A lo menos tres señales apoyan nuestra afirmación. En primer lugar, una de las pocas revisiones sistemáticas al conjunto de la investigación educacional realizada en el país entre 1995 y 2007 revela que del conjunto de los 1300 estudios catastrados, los principales temas de estudio son formación y perfeccionamiento docente (14\%), eficacia y eficiencia educativa (24\%) y pedagogía (14\%) (CIDE y Mineduc, 2007, p. 136). Considerando la amplia dispersión temática que esta revisión presenta, con alrededor de 16 áreas diferentes, destacamos la fuerte presencia numérica de investigaciones sobre formación docente. En segundo lugar, sobresale el aumento de proyectos financiados en el ámbito de la formación de profesores, en comparación con años anteriores. Por ejemplo, en el concurso Fondecyt correspondiente al año 2011, dentro de un total de 22 estudios adjudicados al área educación y pedagogía, vemos que al menos 6 de ellos están directamente vinculados al estudio de la formación docente, lo que además señala una propensión al alza con relación a concursos previos. ${ }^{3}$ Algo similar ocurre con el Fondo Nacional de Investigación en Educación (Fonide) ya que del total de investigaciones financiadas por esta instancia entre los años 2009 y 2011 , cerca del 27\% corresponde solo a esta dimensión específica. Por último, también algunos espacios más recientes de divulgación como congresos o seminarios internacionales avalan esta tendencia; en todos ellos se observa una amplia diversidad de estudios sobre

2 Por ejemplo, la iniciativa Milenio y el apoyo de Conicyt a la creación de centros de investigación que dieron origen al Centro de Investigación Avanzada en Educación (CIAE) y al Centro de Estudios y Políticas Públicas (CEPPE).

3 En este mismo concurso y sobre formación docente, durante los años 2009 y 2010 fueron adjudicados tres y cuatro proyectos, respectivamente. 
formación inicial y continua y un creciente interés por desarrollar y dar a conocer investigaciones en este campo ${ }^{4}$.

Sin embargo, tal como lo decíamos, aunque la preocupación por el trabajo docente y la búsqueda de una formación de calidad es cada vez mayor, llama la atención que la investigación cuyo propósito es estudiar los componentes, procesos y resultados de la formación inicial y continua sea una dimensión menos debatida y profundizada en nuestro país. A pesar del interés, la preocupación, la discusión pública y el número de investigaciones dedicadas al área de la formación docente, carecemos de revisiones, compilaciones o investigaciones sistemáticas y recientes que informen cuáles han sido los intereses y opciones que se materializan en dichos estudios. En otras palabras, aunque tenemos cierta claridad que es un área de estudio en aumento, desconocemos en qué direcciones se han desarrollado esas investigaciones, con qué propósitos y bajo qué rasgos distintivos.

\section{La investigación sobre formación docente. Un campo reciente y singular}

Cada vez más se destaca la estrecha y necesaria relación entre investigación y prácticas de formación docente, convicción que se funda en el creciente desarrollo que ha tenido la investigación en este ámbito. En los últimos treinta años, y cada vez con más fuerza, la literatura teórica y empírica, tanto latinoamericana como de tradiciones de habla inglesa y francófona, reconoce la presencia de algunos rasgos distintivos propios de la naturaleza de las preguntas que se hacen estas investigaciones. Esto ha impulsado que los análisis se trasladen desde el conjunto de la investigación sobre la enseñanza y el profesorado hacia una investigación sobre la formación inicial y continua, que atiende a lo que ocurre en su interior (Cochran-Smith y Fries, 2005; Huberman, 1987; Lenoir y Vanhulle, 2005; Zeichner, 1999). Más aún,

4 La primera versión del Congreso Interdisciplinario de Investigación en Educación presentó aproximadamente 70 investigaciones, de las cuales más de 12 correspondían exclusivamente a formación docente. Ver www.ciie.cl; En el último Encuentro Nacional de Investigadores (ENIN) realizado en 2009 se presentaron más de 20 investigaciones en el campo, entre modalidades conferencia y póster. 
diríamos que este es un campo de estudio relativamente reciente, y que pese a ello ha mostrado en forma paulatina mayor especificidad en sus planteamientos epistemológicos y metodológicos. Así, se dice que la investigación en el campo de la formación de profesores se ha ganado su lugar (Zeichner, 1999) y merece ser explorada en sí misma aunque conservando las relaciones, de cualquier modo ineludibles, con el campo de la enseñanza y el desarrollo profesional docente.

Es así como en algunos países la investigación de la formación docente ha mostrado una historia de rápido desarrollo que la visibiliza dentro del gran paraguas de la investigación educativa (CochranSmith y Fries, 2005; Lenoir y Vanhulle, 2005; Tardif, 2004; Zeichner, 1999). Particularmente en Estados Unidos, encontramos una extensa literatura que permite hablar de una historia de la investigación sobre la formación de profesores. Desde los años cincuenta, cuando emerge como un campo de estudio, hasta nuestros días, donde se la describe a partir de tendencias marcadas por contextos sociales, políticos y científicos (Cochran-Smith y Fries, 2005; Zeichner, 1998b). Destacan también los enfoques y las opciones metodológicas con las cuales aborda su objeto, perfilando una amplia gama de características y diferentes tipos de indagación que la enriquecen y distinguen de otras esferas. Más aún, estos rasgos serían parte de una nueva manera de hacer ciencia y de contribuir a la práctica y a las decisiones políticas ya que, por la naturaleza de su objeto, debe hacerse cargo de la ambigüedad de estar en medio de una investigación "tradicional" orientada a la producción de conocimientos y dentro de una lógica de investigación articulada con el terreno y orientada a la transformación de prácticas (Lenoir y Vanhulle, 2005; Zeichner, 1999).

Lenoir y Vanhulle (2005) plantean que estas características distintivas se originan a partir de tres condiciones que marcan la particularidad de la investigación en este campo: a) investigación y formación se encuentran ubicadas en una misma institución, y en muchas ocasiones son realizadas por el mismo actor (donde se relevan las concepciones que el formador/investigador posee en su doble función); b) dentro de la misma investigación sobre formación docente conviven distintas lógicas distintas: una orientada a la producción de conocimiento, como fin en sí misma, y otra orientada al terreno 
de la enseñanza y la escuela; c) el discurso sobre profesionalización demanda una base de conocimientos que permite constituir la práctica de la enseñanza como una profesión. Por ello la formación exigiría de la investigación un corpus de saberes que constituya un área profesional particular. Si el lector es un investigador en este campo, seguramente podrá reconocerse en alguna de estas condiciones.

Sin embargo, en la medida en que se configura como campo de estudio, la investigación sobre formación docente también ha ido mostrando importantes diferencias acerca de los temas y preguntas legítimos de estudiar en este ámbito. En uno de los principales handbook dedicado a la investigación sobre formación docente en Estados Unidos (Cochran-Smith, Feiman-Nemser, McIntyre, Demers y Association of Teacher Educators, 2008), se destaca cómo la madurez y autonomía que paulatinamente alcanza este grupo de investigaciones se traduce en los distintos caminos que sigue. La variedad de opciones y enfoques al interior de este campo ha sido ampliamente evidenciado por investigaciones que analizan los estudios sobre formación docente realizados durante un cierto periodo de tiempo en países como Brasil, España, Canadá y Estados Unidos, y en regiones como Latinoamérica (André, Simões, Carvalho y Brzezinski, 1999; Cerdán y Grañeras, 1998; Cochran-Smith y Fries, 2005; Kennedy, 1995; Lenoir y Vanhulle, 2005; Messina, 1999; Zeichner, 1999). En todos ellos es posible constatar que distintas conceptualizaciones y discursos sobre la formación docente se materializan en la diversidad de interrogantes que conducen dichos estudios, las que no tienen un carácter único ni uniforme, sino más bien diverso e incluso contradictorio, y delimitan unos problemas legítimos de estudiar que varían entre investigadores. Para comprender las características de la investigación chilena en este campo coincidimos con Cochran Smith y Fries (2005), quienes sugieren que la diversidad de enfoques que la caracterizan puede ser explicada por el modo en que los problemas de la formación del profesor son construidos, estudiados e interpretados. Así, del conjunto de la investigación analizada por estos autores -realizada en las últimas cinco décadas en Estados Unidos- se identificaron al menos tres miradas distintas en el estudio a la formación docente: a) los estudios que conceptualizan la formación como un problema de entrenamiento; b) quienes la estudian como un problema de 
aprendizaje y c) aquellos que la comprenden como un problema de políticas. El hallazgo más interesante de estos autores es que las diferencias entre los objetos de estudio se vincularon a lo que el o los investigadores consideraran como problemático. En suma, este campo de estudio invita a ser explorado con sus propias especificidades. Desconocemos qué caminos recorrerá esta investigación en nuestro país, y las preguntas que se han formulado los investigadores en los últimos años en torno a este campo. Por lo mismo, no tenemos evidencia de cuán diferentes pueden llegar a ser las preguntas que se hacen investigadores de nuestro país en relación con las tendencias internacionales.

\section{¿Qué objetos han sido problematizados por la investigación sobre formación docente en nuestro país en los últimos años?}

Esta pregunta significó para nosotros pensar cuáles serían las áreas temáticas, las características y orientaciones de los objetos que son estudiados en relación con la formación inicial y continua de profesores en Chile. Por esta razón, creemos que aunque sencilla, esta pregunta resulta estratégica. En efecto, en la medida en que tengamos diferentes interrogantes habrá también distintas respuestas $y$, con ello, diversidad de conocimientos sobre el mismo foco de preocupación: la formación docente. Coincidimos con Shulman (1989), en que "para interpretar los resultados de los numerosos estudios es fundamental que el lector entienda las preguntas que se formularon y la manera en que dichas preguntas se situaron, tanto conceptual como metodológicamente" (Shulman, 1989, p. 10). No obstante, pese al incremento sustantivo de estudios que abordan la preparación inicial y continua de los docentes, en nuestro país carecemos de revisiones o análisis sobre la investigación referida a la formación docente que nos permitan caracterizarla de acuerdo con sus principales temáticas, objetos y preguntas abordadas. ¿Qué sabemos de la investigación sobre formación docente realizada en nuestro país en los últimos años? Desconocemos las preguntas y los focos de interés que esta investigación ha tenido, tampoco sabemos si el desarrollo alcanzado presenta rasgos de diversidad $u$ homogeneidad. 
Sostenemos que tanto las preguntas que orientan al investigador como los objetivos que define en la investigación son parte de lo que aquí denominamos "objeto de estudio". En este sentido, el lector podrá advertir que al referirnos a los "objetos de estudio" incluimos las preguntas o problemas que define el investigador, entendido como un foco que se puede identificar en dicha problematización. Por ello, nos referiremos al mismo núcleo conceptual cuando mencionamos la idea de "objeto" "foco problemático" o "preguntas de investigación". En suma, uno de nuestros propósitos fue reconocer las direcciones que ha seguido la investigación sobre formación docente realizada en Chile en relación con las características distintivas de los objetos problematizados durante el periodo $1996-2007^{5}$. Cabe destacar que los hallazgos de esta investigación son acercamientos interesantes a estas preguntas, pero de ninguna manera finalizan la discusión, al contrario, pensamos que son una forma de impulsar nuevas aproximaciones en nuestro país.

\section{Indagar sobre los objetos de estudio. Definiciones metodológicas}

Si lo que perseguíamos era tener una imagen más o menos completa y confiable de los ejes temáticos y los objetos que la investigación sobre formación docente había construido en un periodo de diez años, entonces debimos tomar decisiones atendiendo a las fuentes y el tipo de análisis que utilizaríamos.

¿Qué fuentes entregan información directa sobre el objeto de investigación? Optamos por analizar solo investigaciones concluidas y difundidas por algún medio impreso o digital. Vimos en el resumen de investigación la fuente más accesible y pertinente para hacer un análisis a lo que se investiga en esta área. Dos criterios fundamentan nuestra elección: en primer lugar, el propósito general que orienta la investigación; queríamos conocer los objetos de estudio como

5 Recordamos al lector que este artículo presenta solo una parte de esta investigación. Con el propósito de comprender los procesos de producción de conocimiento en este campo, además intentamos reconocer las condiciones personales contextuales e institucionales que subyacen a la definición de diversos objetos de estudio en el campo de la formación docente. 
un primer paso para comprender cómo este campo se manifiesta y desarrolla en nuestro país tanto desde el punto de vista de sus contenidos como de los procesos de producción de conocimiento en este campo. Dicho fin podía cumplirse sin necesidad de analizar el informe de investigación in extenso. En segundo lugar, consideramos la factibilidad de la ejecución en el acceso al material documental y los tiempos disponibles para efectuar los análisis.

¿Qué investigaciones incluir en la revisión? Reconocemos la limitación inevitable de todo esfuerzo por recoger exhaustivamente el conjunto de la investigación realizada en un país y en un periodo de tiempo. Sabemos que en la medida en que seleccionamos algunas fuentes de publicación estamos excluyendo estudios que no participaron en dichas fuentes. El primer criterio de esta selección es que se traten de estudios difundidos y publicados en algún medio digital o impreso. Para esto, considerando variables de factibilidad, acceso a la información y la presencia de los principales organismos que financian proyectos de investigación, se focalizó la búsqueda en investigaciones apoyadas por el Fondo de Ciencia y Tecnología (Fondecyt), Fondo Investigación y Desarrollo en Educación (Fonide) y el fondo del Consejo Superior de Educación (CSE). Además, asumimos la opción de seleccionar, junto con aquellas financiadas por fondos de prestigio, a todas las investigaciones clasificadas en el área de formación del profesorado, presentadas en los Encuentros Nacionales de Investigación en Educación (ENIN), sin agregar criterios adicionales de rigor, calidad o de publicación en revistas especializadas. Consideramos que aunque esta decisión puede ser arriesgada por cuanto hace más probable encontrarse con investigaciones de menor calidad, nos entregó garantías de incluir a un número importante de investigaciones que de otro modo no habrían sido consideradas, y que dieron una imagen más representativa a nivel nacional de todos los esfuerzos que se realizan por estudiar este campo, más aún tomando en cuenta la precariedad de los espacios para divulgar y hacer circular resultados de investigación en educación en el medio nacional. En suma, revisamos los resultados de los concursos Fondecyt (adjudicados entre los años 1994 y 2005); Fonide (adjudicados entre los años 2006 y 2007); CSE (adjudicados entre 2006 y 2007) y las bases de datos de los Encuentros Nacionales de Investigadores 
en Educación, ENIN (realizados entre 1996 y 2007). Hechas estas precisiones, y luego de elaborar una base de datos exhaustiva sobre los estudios realizados entre 1996 y $2007^{6}$, contamos con un corpus de 82 estudios. Para cada uno de ellos utilizamos el resumen de cada investigación además de otros datos generales de identificación presentes en los reportes.

¿Cómo abordar el análisis de los objetos estudiados? Los criterios para analizar los focos de interés de cada investigación pueden ser muy variados. Ya en otro lugar hemos propuesto al menos tres modelos diferentes para analizar este campo (Cisternas, 2007). Optamos por realizar el análisis de cada resumen de investigación adaptando el modelo utilizado por Lenoir y Vanhulle (2005) en su estudio a la investigación sobre formación docente en Quebec, Canadá. Este modelo organiza los contenidos de investigación a partir de las preocupaciones que levantan los investigadores. Estas preocupaciones se centran en tres polos posibles y las dinámicas que relacionan esos polos. Dichos polos conciernen a los sujetos (a), es decir, los distintos actores de la formación: futuros profesores, formadores y sus competencias, actitudes, representaciones; conocimientos profesionales (b), es decir, disciplinares, pedagógicos, didácticos, sociales: competencias y conocimientos a desarrollar, lugar en los programas, etc.; y dispositivos o procesos de formación (c), es decir, estrategias de formación, estructuras curriculares, modos de construcción de saberes, etc. Cada uno de estos polos puede ser interrogado por sí mismo o respecto de sus relaciones con uno u otro de los restantes polos o con los otros. En esta perspectiva, ampliamos y profundizamos nuestro modelo inicial gracias a los propios datos emergentes. El trabajo inductivo que desarrollamos, encarnado en un proceso de codificación abierta, hizo posible distinguir lo que nosotros hemos denominado como "componentes de la formación", representados por las tres dimensiones ya mencionadas.

6 Incluimos además, dos estudios publicados a comienzos del año 2008. Sin embargo, el corte principal fue de 11 años (1996 y 2007). 


\section{El conjunto de investigaciones analizadas. Desarrollo discontinuo e incipiente}

En primer lugar, proponemos una descripción general del corpus de investigaciones analizadas, sugiriendo tendencias y variables sobre el desarrollo del campo en nuestro país. Una primera caracterización que podemos realizar de este conjunto de investigaciones es la distribución que ha tenido en el periodo estudiado. Para efectos del análisis, los estudios se distribuyeron por bienios (1996-1997; 1998-1999; 2000-2001; 2002-2003, 2004-2005, 2006-2007/8). Como se observa en el gráfico siguiente habría una tendencia al aumento de investigaciones en este campo, particularmente, en el periodo comprendido entre los años 1998 y 2004. Creemos que este momento de "máxima" producción se vincula a la finalización de los Proyectos de Fortalecimiento a la Formación Inicial Docente (PFFID), donde hubo un apoyo económico explícito a las instituciones que participaron para que desarrollaran investigaciones en su área. La curva también evidencia que el interés por estudiar la formación es relativamente nuevo. Probablemente, si retrocedemos unos diez años no sería extraño encontrar un número más reducido de investigaciones en este ámbito.

Gráfico n. ${ }^{\circ}$ 1. Número de investigaciones realizadas en el periodo 1996-2007

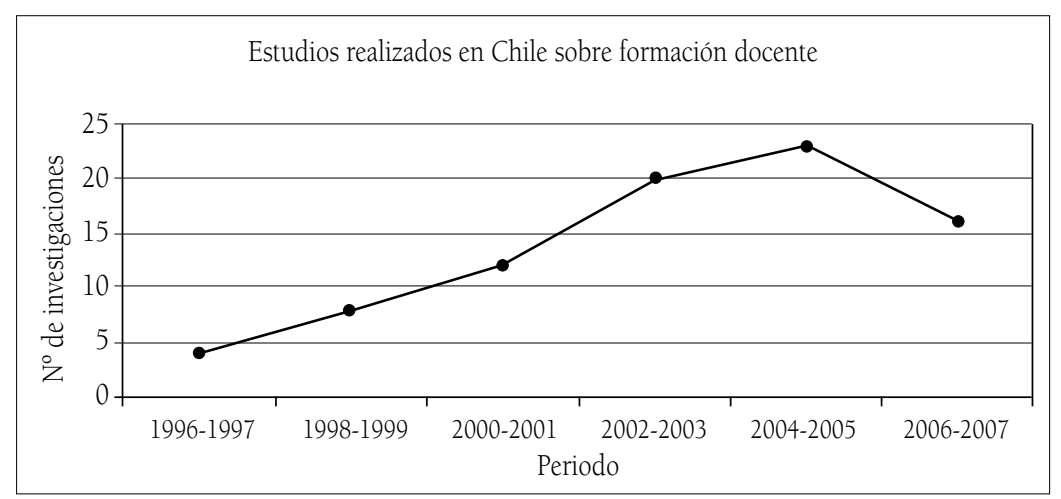

Un segundo aspecto relevante es el financiamiento de las investigaciones sobre formación docente. Como se refleja en la tabla siguiente, si bien se declaran al menos seis fuentes de financiamiento 
diferente, hay una distancia entre quienes acceden a fuentes de financiamiento con prestigio y altos niveles de competitividad (Fondecyt, Fonide, CSE) y fuentes de financiamiento interno, propio de las universidades, y es claro que, al menos en términos de cantidad, el principal apoyo a la investigación sobre formación docente está en las universidades, en desmedro de los fondos concursables ${ }^{7}$. Respecto a este punto, aunque no lo desarrollaremos en este artículo, tenemos hallazgos que aportan algunas explicaciones para comprender las dificultades que tienen los investigadores para diseñar y participar de investigaciones más competitivas y de mayor prestigio.

Tabla n. ${ }^{\circ}$ 1. Modalidades de financiamiento

\begin{tabular}{|l|c|c|}
\hline Modalidades de financiamiento & Número & Porcentaje \\
\hline Fondecyt & 7 & 8,5 \\
\hline Mineduc/Cpeip & 8 & 9,8 \\
\hline Universidad & 45 & 54,9 \\
\hline Fonide & 5 & 6,1 \\
\hline Consejo Superior de Educación & 2 & 2,4 \\
\hline Independiente/autofinanciado & 13 & 15,9 \\
\hline No indica & 2 & 2,4 \\
\hline Total & 82 & 100 \\
\hline
\end{tabular}

Otra característica que podemos deducir del corpus documental estudiado es el número de investigadores que participa en una investigación. Como se refleja en la tabla siguiente, contamos con indicadores promisorios de una práctica de trabajo en equipo paulatinamente instalada entre las investigaciones analizadas. No obstante, también nos llama la atención el importante número de estudios realizados individualmente.

Tabla n. $^{\circ}$ 2. Investigadores participantes por estudio

\begin{tabular}{|l|c|c|}
\hline Investigadores participantes & Número & Porcentaje \\
\hline Un investigador & 25 & 29,8 \\
\hline Dos a tres investigadores & 36 & 45,2 \\
\hline Cuatro o más investigadores & 21 & 25,0 \\
\hline Total & 82 & 100 \\
\hline
\end{tabular}

7 Un dato que ilustra esta condición de financiamiento es que en el concurso Fonide 2007 ningún proyecto ganador orientaba su mirada a este campo. Ver www.mineduc.cl 
Más vinculado al desarrollo de este campo de conocimiento, nos preguntábamos cuál era el espacio que tenían específicamente las investigaciones sobre formación continua. En los últimos años la oferta por perfeccionamiento docente se ha ampliado y actualmente son innumerables las instituciones que ofrecen a los profesores en ejercicio programas de naturaleza y contenidos variados. Al respecto, observamos el predominio contundente de investigaciones que atienden el periodo inicial de la formación de profesores por sobre la formación del profesorado en ejercicio. En definitiva, sabemos mucho menos sobre este tipo de formación, en comparación con la formación inicial.

Tabla n. ${ }^{\circ}$ 3. Formación inicial y formación continua

\begin{tabular}{|l|c|c|}
\hline Tipo de formación de profesores & Número & Porcentaje \\
\hline Formación inicial & 68 & 83 \\
\hline Formación continua & 14 & 17 \\
\hline Total & 82 & 100 \\
\hline
\end{tabular}

Finalmente nos preguntamos sobre qué niveles educativos del profesorado se focaliza la investigación consultada; es decir, si se investigaba sobre la preparación de profesores de enseñanza preescolar, básica, media u otro nivel. Se observan escasas investigaciones que intencionan su mirada hacia distintas pedagogías, sin considerar la naturaleza específica que distingue profesionalmente a un profesor de enseñanza básica, frente a una educadora de párvulos o una docente de educación especial; en su mayoría son estudios a "la formación", sin distinciones. Donde sí hay una clara tendencia a marcar diferencias es entre la formación para profesionales de enseñanza básica y media. También llama la atención el bajo porcentaje de estudios en los niveles de educación preescolar y educación especial.

Tabla n. ${ }^{\circ}$ 4. Formación de profesores según niveles educativos

\begin{tabular}{|l|c|c|}
\hline Nivel de formación de profesores & Número & Porcentaje \\
\hline Educación básica & 21 & 25,6 \\
\hline Educación media & 23 & 28 \\
\hline Educación preescolar & 10 & 12,2 \\
\hline Educación especial & 3 & 3,7 \\
\hline No específica & 25 & 30,5 \\
\hline Total & 82 & 100 \\
\hline
\end{tabular}


En síntesis, existe una tendencia al aumento de investigaciones en el campo, con estudios que se realizan principalmente al alero de las universidades y mucho menos con apoyo de fondos competitivos a nivel nacional. En cuanto a las áreas de investigación en este campo, llama la atención la desproporción que encontramos entre formación inicial y continua y sobre el estudio más específico a la formación de profesores que se desempeñan en distintos niveles del sistema escolar.

\section{¿Qué se estudia de la formación docente? Territorios explorados e inexplorados}

A partir de esta descripción general del corpus, nos detendremos con mayor detalle en las características que presentan los objetos abordados por las investigaciones revisadas. Mostraremos cómo se manifiestan dichos focos de preocupación a través de los actores que participan en la formación; los dispositivos o mecanismos intencionados e institucionalizados que constituyen el currículo diseñado e implementado, y los saberes u objetos de conocimiento que dan contenido a la formación. Estos focos, que Lenoir y Vanhulle (2005) denominan "polos" de la formación, nosotros los hemos llamado "componentes" (aunque nos referiremos también a estos como dimensiones de la formación). Nuestra hipótesis era que nos encontraríamos con unos componentes más estudiados que otros; con unas preguntas más exploradas que otras, que darían cuenta de vacíos en este campo de estudio.

La mirada al conjunto de las investigaciones muestra una constelación de alternativas mucho más limitada de la que esperábamos. Es decir, observamos una tendencia a estudiar ciertos focos problemáticos sobre otros y advertimos la invisibilidad de algunos aspectos de la formación que no parecen ser materia de estudio. ¿Cuál es la diversidad que encontramos al interior de cada componente? Es decir, ¿qué actores, dispositivos de formación y conocimientos profesionales son objeto de estudio en el corpus analizado? La siguiente tabla presenta la frecuencia de estudios que indaga sobre cada uno de estos aspectos. Se debe considerar que una misma investigación puede abordar más de un componente. Desglosaremos cada uno de ellos a continuación. 
Tabla n. ${ }^{\circ}$ 5. Componentes estudiados de la formación docente

\begin{tabular}{|l|l|c|c|}
\hline \multicolumn{2}{|c|}{} & \multicolumn{2}{c|}{ Tendencia } \\
\cline { 2 - 4 } \multicolumn{2}{|c|}{ Componentes estudiados } & Frecuencia de aparición & Porcentaje \\
\cline { 2 - 4 } & DISPOSITIVOS & 45 & $45,5 \%$ \\
\cline { 2 - 4 } & CONOCIMIENTOS PROFESIONALES & 39 & $39,4 \%$ \\
\hline Total & & 15 & $15,2 \%$ \\
\hline
\end{tabular}

\section{Preguntándose por los actores relevantes en el proceso de formación}

Los actores implicados en el proceso de formación inicial y continua son múltiples, e incluyen tanto a quienes participan en el diseño, implementación, apoyo y evaluación de la formación como a quienes reciben o se benefician de esta. En el caso de las investigaciones realizadas en Chile nos preguntamos ¿qué actores son parte de la problematización sobre formación docente?; ¿en quiénes pone su atención el ojo del investigador?, ¿a quienes olvida?

Dentro de los componentes de la formación estudiados, este es el más desarrollado. Del corpus analizado, un 66\% (54 investigaciones) pone su atención sobre cinco actores relevantes: estudiante de pedagogía, formador de profesores, profesor en ejercicio, recién egresado, profesor guía de prácticas ${ }^{8}$.

Gráfico n. ${ }^{\circ}$ 2. Actores de la formación docente estudiados

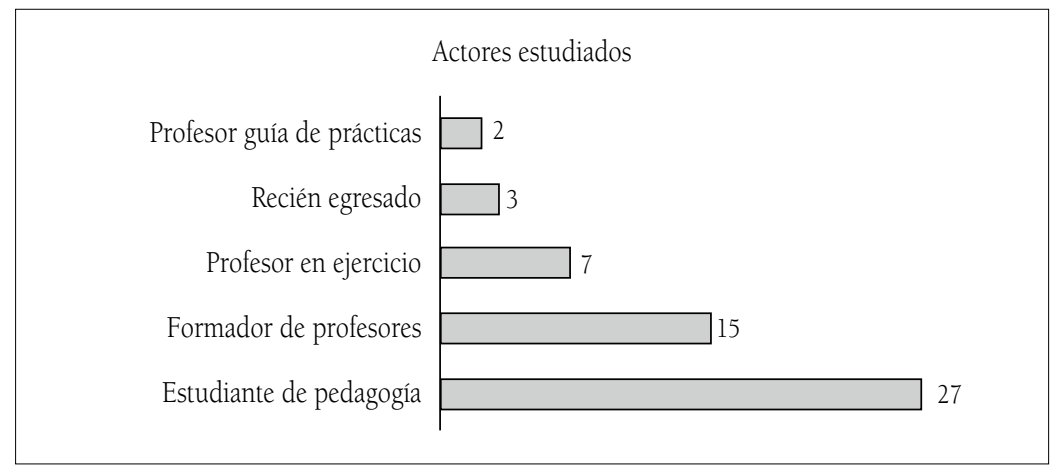

8 Recordemos que un mismo estudio puede formularse en atención a más de un actor o más de un dispositivo. 
Si observamos el gráfico anterior se puede apreciar que las investigaciones conceden una atención desproporcionada entre los que son "sujetos de la formación", estudiantes de pedagogía o profesores en ejercicio, en comparación con los "formadores", aquellos que diseñan e implementan la formación. A continuación describimos cómo se materializan estas diferencias.

a) Estudiante de pedagogía y profesor en ejercicio. Una mirada desigual

En las investigaciones predomina un interés por el estudiante de pedagogía en su proceso de formación inicial por sobre los egresados y el profesor en ejercicio. Al primero se lo estudia desde una amplia diversidad de perspectivas mientras los segundos son considerados principalmente para evaluar o validar dispositivos diseñados casi siempre por los propios investigadores-formadores.

En el primer caso, las investigaciones se interesan por el estudiante de pedagogía en distintos momentos de su formación: cuando comienza o finaliza sus estudios o en semestres específicos. También, encontramos estudios que se focalizan en estudiantes que cumplen con ciertos requisitos de la formación: estar realizando la práctica profesional; haber participado de un curso en específico o poseer alguna característica particular (por ejemplo, determinado puntaje de ingreso, origen étnico, estilo de aprendizaje). ¿Qué propiedades del actor son investigadas cuando se trata del estudiante de pedagogía? Principalmente, se problematiza para describir o caracterizar sus saberes, habilidades, actitudes, competencias específicas o para conocer sus valoraciones en distintos periodos de su formación. Menos frecuentes son las investigaciones que seleccionan al estudiante de pedagogía para conocer sus procesos de aprendizaje o la forma en que se convierte en profesor.

El profesor en ejercicio tiene un lugar mucho menos protagónico dentro del conjunto de investigaciones. Esto parece explicarse por los datos que diéramos al inicio: la formación continua ha sido un área explorada en menor grado en comparación con la formación inicial. Sin embargo, esta desatención también reflejaría 
una dimensión negada: el profesor en ejercicio no parece ser un actor relevante para la formación inicial; sus dificultades una vez egresado, los conocimientos construidos en la práctica, los contextos escolares donde se inserta, las necesidades de formación que emergen de esas experiencias, no constituyen una fuente que, de ser indagada, pudiese nutrir la formación en pregrado, y los programas de formación continua. Las investigaciones se interesan por este actor como un "receptor de formación", en este caso de perfeccionamiento docente o, cuando más, como un informante primordial para el diseño o evaluación de dichas experiencias formativas, que en su mayoría impulsa el propio investigador en su función de formador de profesores.

En esta misma línea, otro actor que recibe atención marginal (solo un 7\% de las 56 investigaciones que atienden en este componente) es el profesor recién egresado. En las pocas oportunidades donde se lo define como objeto de estudio lo diferencian claramente del estudiante de pedagogía y del profesor en ejercicio ${ }^{9}$. En efecto, se busca que evalúe su formación y declare fortalezas y debilidades a partir de las demandas del contexto profesional. Ni sus características, ni los procesos de inserción a la docencia (salvo un estudio), ni sus prácticas pedagógicas y cómo estas van transformándose, son parte de las preguntas que la investigación en este campo ha podido responder. Sin embargo, en los últimos años los procesos de acreditación y algunas investigaciones específicas han impulsado la mirada hacia este actor tan relevante. Aunque nuestra revisión solo alcanza los estudios publicados hasta el año 2007, y este panorama podía haber cambiado en los últimos años, consideramos que el dato es un antecedente en sí mismo de gran importancia, pues denuncia la invisibilidad que durante años ha tenido este actor para la investigación sobre formación de profesores.

9 Excluimos las investigaciones que aunque incluían una muestra de profesores recién egresados, lo hacían solo como parte de la estrategia metodológica y no desde la definición del objeto y propósitos. Es decir, cuando el actor egresado de pedagogía no era parte del problema en estudio. 


\section{b) Formador de profesores, un actor difuso y poco estudiado}

El segundo foco de interés mostrado en las investigaciones analizadas se ocupa de quienes cumplen el rol de formar profesores tanto en el inicio como en instancias de formación continua. Cabe destacar la significativa diferencia entre esta categoría y la de los estudios dedicados al estudiante de pedagogía. Se estudia un 50\% menos al formador que lo que se hace con el futuro profesor.

¿Qué se mira del formador?, ¿cuáles son las traducciones que hace la investigación frente a este actor?, lo cierto es que no muchas, al contrario, se conceptualiza al formador como parte de un escenario que requiere ser caracterizado de modo similar a como se hace con el estudiante de pedagogía. Es decir, interesa describir las percepciones, creencias, conocimientos específicos, representaciones del formador sobre áreas disciplinares, enfoques de enseñanza, expectativas y valoración del aprendizaje de sus alumnos (estudiantes de pedagogía). Excepcionalmente se plantean investigaciones que informen sobre los procesos de reflexión, transformación o aprendizaje de estos actores. De hecho, los estudios que miran un formador de profesores "genérico", es decir, pertenecen a una unidad académica o a una universidad y rara vez a un área de la formación más específica. Llama la atención que no veamos una diversidad mayor dentro de este grupo; pareciera que el formador es una categoría homogénea, sin especificidades como las que muestra el estudiante de pedagogía. Sin desconocer lo común que atraviesa a todo perfil de un formador, sabemos también que hay especificidades. Los conocimientos y capacidades que pone en acción un formador responsable de la formación de especialidad (por ejemplo, las didácticas específicas) pueden ser de naturaleza distinta en comparación con un formador responsable del área pedagógica (por ejemplo, las teorías de aprendizaje). En definitiva, el formador de profesores en su dimensión compleja y diversa permanece invisible en nuestro país.

\section{Los dispositivos: ¿cuáles son los mecanismos de formación estudiados?}

Al comienzo de esta investigación sostuvimos que buena parte de la esencia de una acción de formación se encuentra en los dispositivos. 
Si entendemos que la formación docente está compuesta por acciones instrumentadas con carácter intencionado, entonces este foco de problematización resulta imprescindible en una mirada sobre la formación docente. En nuestro análisis, los dispositivos son todas aquellas estructuras, ordenamientos, estrategias o herramientas que forman parte de la formación: desde dispositivos generales como son el propio currículo de formación hasta estrategias específicas como cursos, instrumentos, estrategias de enseñanza-aprendizaje. En esa perspectiva (y con estas expectativas) revisamos el conjunto de investigaciones preguntándonos ¿qué dispositivos estudia la investigación sobre formación de profesores en nuestro país?, ¿cuáles son sus principales preocupaciones?, ¿de qué manera se aproxima a ellos?

El proceso inductivo de codificación desarrollado en esta investigación generó menos diversidad de la que esperábamos en comparación con los recorridos que habíamos visto de otros países sobre este mismo campo de estudio. El siguiente gráfico ilustra las áreas que han sido objeto de mayor preocupación en esta dimensión. Pasaremos a revisarlas por separado.

Gráfico n. ${ }^{0}$ 3. Dispositivos de la formación docente estudiados

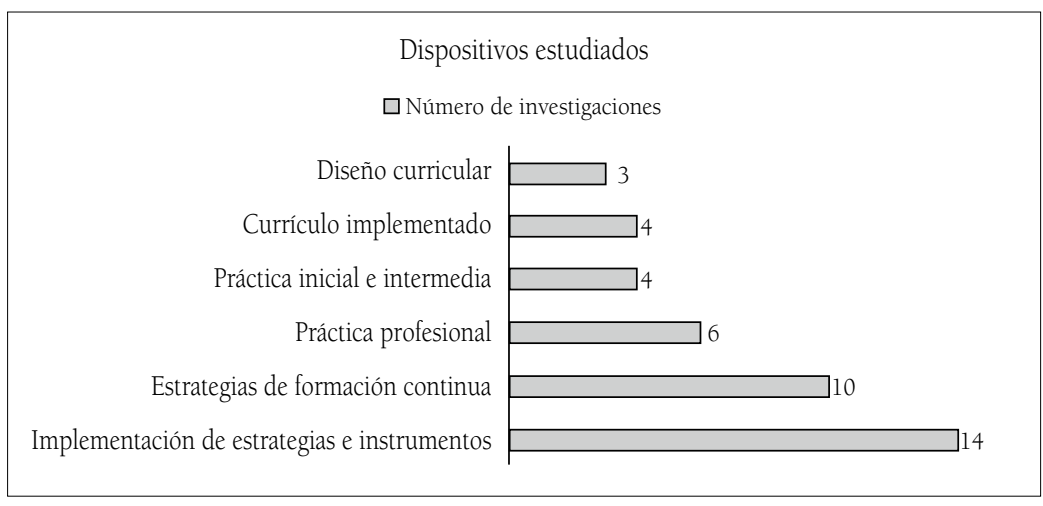

a) Investigando la propia práctica de formación inicial

El principal dispositivo que resulta ser objeto de indagación en este campo no es uno, sino un conjunto heterogéneo (en modalidad, alcance y propósito) de estrategias de formación fuertemente 
contextualizadas en programas específicos de formación inicial. Son, además, estudios con una característica adicional: en todas ellas el investigador es también formador y convierte en objeto de estudio su propia práctica pedagógica. Se trata entonces de dispositivos específicos que apuntan a propósitos diferentes: unas intentan describir el proceso, otras pretenden evaluarlas. Sin embargo, el principal interés está en documentar el proceso, es decir, implementar experiencias o estrategias que se escogen por ser consideradas como formas de innovar al interior de cursos específicos o líneas de formación. El segundo grupo de investigaciones, de menor presencia, busca testear, evaluar o comprobar el funcionamiento de algunas estrategias (por ejemplo, tipos de acompañamiento a la práctica, técnica de conversación reflexivo-crítica) o de herramientas (como talleres, portafolio, guía de reflexión). Algunos estudios realizan esta evaluación a partir de las percepciones de los estudiantes de pedagogía, participantes de la estrategia y otros mediante procedimientos de pre y post-test.

b) Prácticas iniciales, intermedias y profesionales: ¿una misma práctica?

Un primer aspecto para destacar es la mirada marginal que presenta el que podríamos considerar como uno de los dispositivos centrales para la formación inicial. Contamos con evidencias múltiples sobre la importancia que tienen las distintas instancias prácticas para el aprendizaje docente, la construcción de conocimiento práctico anclado y sustentado por la teoría, y la formación de un habitus reflexivo que fortalezca el ejercicio autónomo de la profesión. Sin embargo, dentro del total de investigaciones solo un $10 \%$ delimita su objeto de estudio en este componente. Más aún, al igual que con el formador de profesores, la categoría "práctica" es estudiada casi exclusivamente como práctica profesional o terminal. Más específicamente, en ella se busca: conocer el impacto de la práctica sobre algunas características profesionales (autoestima, seguridad, capacidad reflexiva) de los futuros profesores; establecer lo que hacen los distintos responsables de este proceso y describir los problemas habituales en esta instancia formativa. Solo un par de excepciones también atiende a las prácticas iniciales (todas aquellas que se realizan antes de la práctica terminal) 
y buscan documentar algunas experiencias de acompañamiento. ¿Cómo se aborda la indagación de estas prácticas? a) desde sus principales características, modalidades y funcionamiento; b) de la autopercepción de capacidades desarrolladas por los actores involucrados (en particular, los estudiantes de pedagogía); c) como dificultades reconocidas por distintos actores.

Nuevamente, como lo hemos visto en otros componentes estudiados, el objeto "práctica" se presenta de forma difusa y uniforme. Las investigaciones invisibilizan los diferentes propósitos, contenidos y modalidades de intervención (el estudiante de pedagogía no hace lo mismo en una práctica de observación en primer año o en una de aplicación didáctica en tercer año de la formación). Tampoco se muestran estudios que aporten conocimientos sobre cómo se aprende en las prácticas.

\section{c) Características de programas y estrategias de formación continua}

En el caso de la formación continua persiste la tendencia de mostrarse como un foco de estudio desatendido dentro de todo el conjunto de investigaciones sobre formación docente. Todas las investigaciones delimitan su objeto a un programa específico. Estos programas poseen distinta envergadura, pero en su mayoría están diseñados por universidades (diplomados, postítulos, cursos presenciales o virtuales) o por equipos de investigadores que diseñan estrategias o modelos de capacitación. También, se observa el estudio de acompañamientos (o asesoramientos) a docentes originados en programas como Mece o Liceo para Todos (talleres de reflexión entre pares, grupos de aprendizaje docente). El objeto en estos casos se construye a partir del diseño, aplicación y evaluación de propuestas formativas. ¿Qué se mira de estos dispositivos? En primer lugar, se los pone en relación explícita con unos conocimientos profesionales (didáctica de las matemáticas; evaluación auténtica; gestión de la convivencia en el aula; alfabetización científica, entre otros) y en algunas ocasiones se los relaciona también con el profesor en ejercicio como actor que se apropia de los saberes que transmiten estos programas de formación. Así, se construyen objetos de estudio que intentan dar cuenta de 
una relación entre dispositivo-conocimiento-actor (relación que se muestra más nítida en comparación con los estudios referidos a la formación inicial), algo que podría estar reflejando una comprensión más amplia del aprendizaje docente. Además, sostienen una búsqueda por conocer el impacto de estos programas que muchas veces va de la mano con el conocimiento de los procesos de aprendizaje docente generados en esas instancias de formación.

\section{Los conocimientos profesionales: ¿cuál es el contenido de la formación?}

Pensar en este componente supone detenerse en el contenido que encarnan los dispositivos de formación. ¿Cuáles son los conocimientos profesionales que distinguen a la profesión docente de otras profesiones?, ¿qué deben aprender los futuros profesores y, más tarde, qué competencias deben actualizar? Esta dimensión ha resultado ser la menos explorada dentro del conjunto revisado. La problematización de la formación del profesorado aparece mucho más vinculada a quiénes y cómo se forma y mucho menos a qué se debe aprender en el proceso de convertirse en profesor.

Las propias investigaciones analizadas fueron dibujando tres tipos de saber profesional que conceptualizamos utilizando la división en áreas de formación que, con pequeñas modificaciones, realizan las carreras de pedagogía en nuestro país para organizar su plan de estudios (CNAP, 2007). De este modo, distinguimos aquellas investigaciones que ponen atención sobre conocimientos vinculados a las áreas de formación pedagógica, formación general y formación en la especialidad. En este contexto, observamos que solo un $12 \%$ (diez investigaciones) del total de estudios revisados problematiza la formación atendiendo a alguno de estos tres componentes. Cabe precisar que buscábamos aquellas investigaciones que se interesaban directa y explícitamente por este componente de la formación, ya sea en relación con los actores o los dispositivos. 
Gráfico n. ${ }^{\circ}$ 4. Conocimientos profesionales de la formación docente estudiados

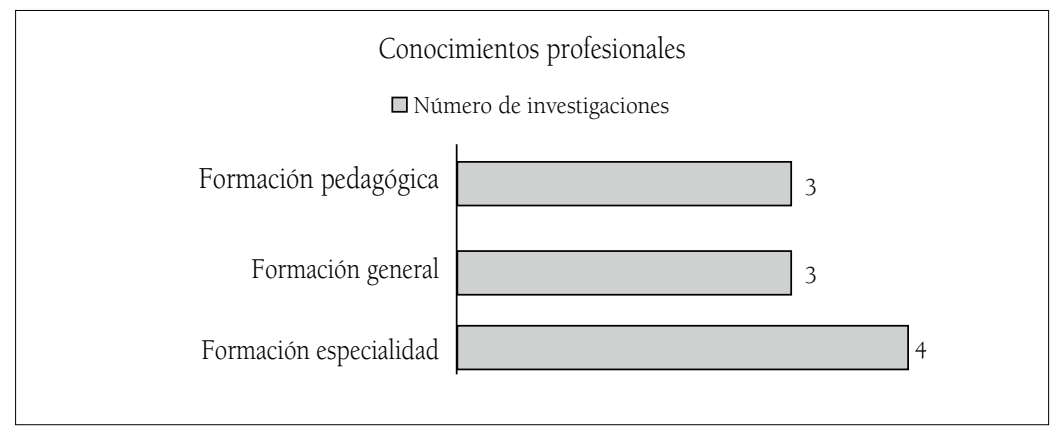

En términos específicos, los objetos estudiados en relación con el área de formación general comprenden competencias asociadas al reconocimiento de los factores sociales y culturales que intervienen en los procesos educativos y actitudes éticas inherentes a la relación profesional entre pares y la responsabilidad por los educandos. El área formación pedagógica aborda fundamentalmente conocimientos sobre procesos de aprendizaje y desarrollo y conocimientos del proceso de enseñanza y organización del currículo y la enseñanza, el sentido y propósito de diversas estrategias de enseñanza y evaluación, el conocimiento y práctica de la pedagogía de la disciplina o especialidad, creación y manejo de ambientes de aprendizaje. Por último, la formación en la especialidad involucra el estudio de los saberes asociados a las áreas de contenido que requieren de apropiación por parte de los docentes con el fin de enseñar eficientemente los contenidos.

A modo de síntesis presentamos una tabla que incluye el número de investigaciones asociadas a cada uno de los componentes específicos que hemos descrito hasta ahora: 
Tabla n. ${ }^{\circ}$ 6. Componentes de la formación

\begin{tabular}{|c|c|c|}
\hline \multicolumn{3}{|c|}{ Componentes de la formación } \\
\hline Actores & Dispositivos & $\begin{array}{l}\text { Conocimientos } \\
\text { profesionales }\end{array}$ \\
\hline $\begin{array}{c}\text { Estudiantes de pedagogía } \\
\text { (27) }\end{array}$ & $\begin{array}{l}\text { Estrategias instrumentos de } \\
\text { formación inicial (14) }\end{array}$ & Formación general (3) \\
\hline $\begin{array}{c}\text { Formador de profesores } \\
(15)\end{array}$ & $\begin{array}{l}\text { Estrategias de formación } \\
\text { continua (10) }\end{array}$ & $\begin{array}{l}\text { Formación de especialidad } \\
\qquad \text { (4) }\end{array}$ \\
\hline Profesor en ejercicio (7) & Práctica profesional (6) & Formación profesional (3) \\
\hline Egresado/principiante (4) & $\begin{array}{c}\text { Prácticas } \\
\text { Inicial/intermedia (4) }\end{array}$ & \\
\hline
\end{tabular}

\section{¿Uno o más componentes? Débil acercamiento a la complejidad de la formación docente}

En un segundo nivel de análisis, nos preguntamos por el grado de interacción de estas tres dimensiones en cada estudio. Es decir, si los problemas formulados se definían a partir de un componente en particular o poniendo en diálogo distintas dimensiones. Observamos que algunos casos construyen el problema a partir de un componente y otros complejizan y amplían el objeto indagando sobre la interacción entre dos o más componentes de la formación. Si bien nuestra visión inicial (quizá algo ingenua y sin antecedentes previos que permitieran anticiparnos) nos hizo creer que la mayoría de las investigaciones abordarían solo un componente de la formación, en el proceso de análisis constatamos que una misma investigación podía transformar dos componentes en objeto de investigación. Esta diferencia puede conceptualizarse como dos modos de construir el objeto de estudio en el campo de la formación docente: mientras unos objetos (la mayoría, $58 \%$ ) se construyen de manera unidimensional otros lo hacen en una perspectiva pluridimensional (39\%).

En efecto, en el primer caso observamos que la mayoría de las investigaciones consultadas opta por indagar sobre uno de los tres componentes de la formación. Más aún, como lo refleja la tabla n. ${ }^{\circ} 6$, son los actores del proceso formativo los que concentran 
la mirada de los investigadores: se preguntan por las habilidades, creencias o conocimientos de estudiantes de pedagogía, profesores en formación y/o formadores de docentes. Mientras que cuando se indaga sobre los dispositivos de la formación, se describen y/o comparan actividades específicas, diseños curriculares o programas de cursos. En ambos casos, insistimos, se focaliza el problema en una dimensión de la formación.

En el segundo grupo de investigaciones, cuando se estudian dos o más componentes de la formación, estas conectan esas dimensiones de dos maneras diferentes. Un grupo mayoritario investiga unos actores específicos en relación con unos dispositivos de formación: en casi todos estos casos se indaga sobre la valoración que hace el docente de unas modalidades de formación o bien se estudia el impacto que un dispositivo alcanza sobre los saberes, prácticas y/o creencias de un actor. Por ejemplo, se problematiza la relación entre unas características de las prácticas profesionales (dispositivo) y unas habilidades adquiridas por el futuro profesor (actor) o bien se apunta a las actividades de un curso de formación continua (dispositivo) y cómo este fue percibido por los profesores beneficiados (actor). El otro tipo de conexión o manifestación de esta pluridimensionalidad se da cuando unos dispositivos se relacionan con conocimientos en áreas de especialidad y pedagógica. Por ejemplo, se diseña una estrategia específica de formación continua (dispositivo) para el aprendizaje de unos procedimientos evaluativos (conocimiento pedagógico).

Por otra parte, si comparamos las investigaciones sobre formación continua con las de formación inicial, vemos que pese al reducido número de las primeras, observamos un mayor acercamiento a la complejidad en el estudio a la formación, seleccionando más de un componente en la construcción de sus objetos. Por ejemplo, un estudio que diseña, implementa y evalúa una estrategia de acompañamiento a docentes para el aprendizaje de estrategias didácticas en ciencias. Comparativamente, estas investigaciones aunque muy escasas dentro del conjunto revisado, destacan por construir un objeto complejizado y pluridimensional de la formación docente, frente a opciones unidimensionales del fenómeno, mucho más presentes en los estudios a la formación inicial. 


\section{Lo que no se deja ver en los estudios acerca de la formación docente en Chile. Nuevas preguntas para seguir investigando}

A modo de conclusión, pensamos que el ejercicio de analizar lo que se ha investigado en el campo de la formación docente en Chile entregó pistas valiosas para comprender el estado actual del conocimiento que tenemos sobre un área tan estratégica. Detenernos a reflexionar sobre aquello que aún no se logra mirar, eso que aún no nos preguntamos (y por tanto, no convertimos en objeto de estudio) contribuye a repensar no solo en los focos de preocupación, sino también en las prioridades de financiamiento y el inicio, desarrollo y consolidación de líneas de investigación que tributen nuevos conocimientos para la formación docente en Chile. Así, frente a las preguntas ¿cuáles son los objetos de estudio que menor presencia tienen dentro de nuestro corpus analizado?, ¿cuáles son esos territorios sin explorar aún?, este mapa sinóptico elaborado a partir del análisis a 82 investigaciones, nos permite aproximar algunas respuestas.

En comparación con la formación inicial, sabemos mucho menos sobre la formación continua de los profesores en Chile. Desconocemos la naturaleza de la formación y el aprendizaje en profesores en ejercicio ¿Qué enfoques de formación continua predominan en nuestro país?, ¿cómo se conceptualiza la formación en estas instancias?, ¿qué se aprende allí?, ¿cómo se definen las necesidades de aprendizaje?, ¿de qué manera participan los profesores en estas definiciones? En este sentido, es escasa la problematización sobre las características y modalidades que predominan (cursos, talleres, diplomados, postítulo, asesorías, comunidades de aprendizaje, etc.) ni en qué tiempos y formas se imparten (presencial, a distancia, virtual; por semanas, meses, semestres). Menos aún existen estudios que indaguen sobre el impacto en los saberes y prácticas que esta formación alcanza sobre los profesores. Pese a ello, sí sabemos un poco más gracias al estudio de algunos programas específicos de formación continua. Observamos interesantes señales (aunque minoritarias dentro del conjunto) que muestran investigaciones indagando los nexos entre unas estrategias de formación, los contextos profesionales y los conocimientos y prácticas que estas 
propician, y con ello, asumiendo la complejidad en el estudio de la formación y el aprendizaje docente.

Los silencios de la investigación en este ámbito se refuerzan con la escasa atención puesta sobre el profesor en ejercicio, como un actor que puede informar y nutrir tanto a la formación inicial como a la formación continua. Y observamos un reducido interés por los conocimientos que construye este profesor en las distintas instancias de formación continua. De hecho, pocos caracterizan sus prácticas de enseñanza relacionándolas con su formación, ni tampoco observamos investigaciones que indagan en las conexiones existentes entre la formación inicial y la formación continua.

Otra dimensión aún invisible es el formador de profesores. Si bien este actor es el segundo en presencia dentro de todos los que atienden las investigaciones, observamos que se conceptualiza a un formador que hemos denominado "genérico", y por lo mismo, sostenemos que es un actor estudiado desde aspectos muy generales. En nuestro análisis dentro de esta gran categoría de formador de profesores se ignora a un actor diverso en responsabilidades formativas y conocimientos cuyas especificidades pueden verse, por ejemplo, mediante las áreas de formación que comprenden las carreras de pedagogía. Por otra parte, se produce conocimiento sobre el formador casi exclusivamente desde sus concepciones sobre aprendizaje, la valoración que este hace de algún aspecto de la formación o las creencias o conocimientos respecto de un tema. Sin embargo, aunque las hay, son pocas las investigaciones que dan pistas sobre las prácticas del formador de profesores y menos aún las interacciones entre determinadas acciones formativas y los aprendizajes que alcanza el profesor en formación.

En tercer lugar, las indagaciones sobre experiencias prácticas dentro de la formación inicial son menos valoradas como problemáticas de investigación en cuanto a mostrar los procesos involucrados en dichas prácticas y la manera en que estos dispositivos impactan sobre el aprendizaje del futuro profesor. Llama la atención que se pregunten muy poco por lo que ocurre durante las prácticas iniciales e intermedias, y focalicen los problemas investigados en la práctica 
final o profesional. Creemos que, como en el caso de los formadores, la "práctica profesional" es problematizada de manera tan amplia que impide profundizar nuestra comprensión sobre la diversidad de propósitos, contenidos y actividades de esta trascendental área de la formación. Más aún, vemos que se trata de una omisión difícil de comprender en un contexto donde casi todas las instituciones formadoras de nuestro país han implementado las denominadas prácticas progresivas, reconociendo la importancia que estas tienen para la construcción de competencias docentes.

Los aprendizajes, las prácticas, las dificultades del egresado de pedagogía, nos parece una de las omisiones más preocupantes y de mayores consecuencias dentro del conjunto de objetos invisibilizados por la investigación nacional en este campo. El estudiante que recibe una formación inicial y que luego de cumplir su periodo formativo egresa con un título que lo habilita para desempeñar su profesión resulta ser un actor marginal si se lo compara con los otros actores investigados y dentro del conjunto total de investigaciones ¿Qué sabemos de él?, ¿qué impacto formativo tuvo la formación inicial en ese egresado?, ¿cómo lo que aprendió se transforma en el ejercicio real y contextualizado de la profesión? Más se tensiona esta ausencia si atendemos al impulso que ha tenido en los últimos años temas como la inducción a la docencia, las mentorías y el seguimiento a los egresados.

Asimismo, los conocimientos profesionales que encarnan la formación docente son los menos indagados dentro de los tres componentes de la formación que han sido explorados ¿Qué debe saber el futuro profesor?, ¿cuál es la naturaleza de ese saber?, ¿cómo se aprenden esos saberes?, son preguntas excepcionales dentro de la revisión que hicimos. Los estudios existentes dividen esta discusión entre áreas de conocimiento pedagógico, de la especialidad y generales. Sin embargo, las investigaciones que efectivamente se ocupan de esta dimensión muestran señas de atender a una construcción más amplia del problema de la formación, interrogando las relaciones entre unos actores, esos conocimientos y las estrategias de formación. 


\section{La reflexividad acerca de lo investigado: un ejercicio difícil pero necesario}

Para finalizar, queremos destacar la naturaleza de nuestra pregunta y la intención que había tras ella cuando formulamos esta investigación. Preguntarse por los caminos que han sido recorridos por las investigaciones y cuáles aún permanecen sin explorar es una forma, pensamos, de impulsar el desarrollo de un campo de conocimiento en particular, ya que convoca a investigadores e investigadoras al análisis reflexivo y crítico de la investigación que nosotros mismos producimos.

En efecto, creemos que la madurez de un campo de conocimiento está fuertemente relacionada con la capacidad reflexiva que este tenga sobre sí mismo. Estamos convencidos de que la elección también implica renuncia. Por ello, reconocer, revisar, evaluar y debatir qué estamos investigando permitiría tensionar lo que se selecciona como legítimo en relación con lo que se omite o excluye de los ojos del investigador. En definitiva, la formación y el aprendizaje de los profesores de nuestro país se enriquecerían si le diéramos un impulso a este campo de estudio desde una perspectiva crítica y reflexiva de nuestras propias prácticas investigativas.

\section{Referencias bibliográficas}

André, M.; Simões, R.; Carvalho, J. y Brzezinski, I. (1999) Estado da Arte da Formação de Professores no Brasil. Educação y Sociedade, 20(68), 301-309.

Araújo-Oliveira, A. (2009) Desafios e obstáculos da pesquisa em educação para a transformação das práticas pedagógicas. Educação Revista do Centro de Educação UFSM, 34, 617-632. Recuperado el 20 marzo 2010

Araújo-Oliveira, A.; Lenoir, A. y Lebrun, J. (2009) Étude crítique de la documetation scientifique brésilienne relative à l'analyse des practiques enseignantes. Canadian Journal of Education, 32(2), 285-316. Recuperado el 20 de marzo de 2010, de http://www.csse-scee.ca/CJE/ Articles/CJE32-2.html

Arzola, S. y Lenoir, I. (2009) Las prácticas de enseñanza: una mirada transversal. Pensamiento Educativo, 44-45, 7-29. 
Ávalos, B. (1997) Investigación, políticas y prácticas. El caso de la formación inicial de docentes. Pensamiento Educativo, 20, 437-455.

Borko, H.; Whitcomb, J. y Kathryn, B. (2008) Genres of Research in Teacher Education. En M. Cochran-Smith, S. Feiman-Nemser, D. McIntyre y K. Demers (Eds.), Handbook of Research on Teacher Education Enduring Questions in Changing Contexts (3 ed., pp. 1017-1045). New York: Routledge.

Cerdán, J. y Grañeras, M. (1998) La investigación sobre formación del profesorado. En J. Cerdán y M. Grañeras (Eds.), La investigación sobre profesorado II 1993-1997 (pp. 41-60). Madrid: CIDE.

CIDE y Mineduc (2007) Estado del arte de la investigación y desarrollo en educación en Chile (Versión Digital). Santiago: Mineduc.

Cisternas, T (2007) Consideraciones para el análisis de la investigación sobre formación docente en Chile. Pensamiento Educativo, 41(1), 189-206

CNAP (2007) Criterios de evaluación de carreras de educación. Recuperado el 15 de marzo de 2011, de http://www.cnachile.cl/criterios-especificospara-carreras/

Cochran-Smith, M. (2001) The Outcomes Question in Teacher Education. Teaching and Teacher Education, 17, 527-546.

Cochran-Smith, M. (2002) The Research Base for Teacher Education. Metaphors We Live (and die?) by. Journal of Teacher Education, 53(4), 283-285.

Cochran-Smith, M. y Demers, K. E. (2008) How Do We Know What We Know? Research and Teacher Education. En M. Cochran-Smith, S. Feiman-Nemser, D. McIntyre y K. Demers (Eds.), Handbook of Research on Teacher Education Enduring Questions in Changing Contexts (3 ed., pp. 1009-1016). New York: Routledge.

Cochran-Smith, M.; Feiman-Nemser, S.; McIntyre, D.J. y Demers, K. E., y Association of Teacher Educators (2008) Handbook of Research on Teacher Education: Enduring Questions in Changing Contexts (3rd ed.). New York: Routledge.

Cochran-Smith, M. y Fries, K. (2005) Researching Teacher Education in Changing Times: Politics and Paradigms. En AERA (Ed.), Studying Teacher Education: the Report of the AERA Panel on Research and Teacher Education (pp. 69-109). New Jersey: Lawrence Erlbaum Associates.

Cochran-Smith, M.; Fries, K. y Zeichner, K. (2006) Estudio sobre la formación del profesorado en los Estados Unidos: descripción del informe del comité de la American Educational Research Association (AERA) sobre 
investigación y formación del profesorado. Revista de Educación, 340, 87-116.

Comisión Formación Docente (2005) Informe Comisión sobre Formación Inicial Docente. Santiago, Chile.

Comisión Formación Docente (2007) Informe Sintesis Tercer Encuentro Nacional de Formación Docente Políticas de Formación Docente en los nuevos escenarios. Santiago, Chile.

Huberman, M. (1987) La recherche sur la formation: Quelques hypothèses provocatrices. Recherche et Formation, 1, 11-25.

Kennedy, M. (1995) Research Genres in Teacher Education. En F. Murray (Ed.), Knowledge Base in Teacher Education (pp. 120-152). Washington, D.C.: McGraw Hill.

Lara, M.; Förster, C. y Gorichon, S. (2007) Transferencia de la investigación educacional a la formación inicial de profesores. Calidad en la Educación, $27,178-204$.

Lenoir, I. y Vanhulle, S. (2005) L'état de la recherche au Québec sur la formation à l'enseignement: vers de nouvelles perspectives. Sherbrooke (Canada): Editions du CRP, Université de Sherbrooke.

Lenoir, Y. (2006) Pesquisar e formar: repensar o lugar e a função da prática de ensino. Educação y Sociedade, 27(97), 1299-1325.

Messina, G. (1999) Investigación en o investigación acerca de la formación docente: un estado del arte en los noventa. Revista Iberoamericana de Educación, 19, 145-207.

MINEDUC (2006) Informe Consejo Asesor Presidencial para la Calidad de la Educación. Santiago, Chile: Ministerio de Educación

OCDE (2004) Revisión de políticas nacionales de educación. Santiago, Chile.

Shulman, L.S. (1989) Paradigmas y programas de investigación en el estudio de la enseñanza: Una perspectiva contemporánea. En M. C. Wittrock (Ed.), La investigación de la enseñanza: enfoques, teorías y métodos. (pp. 9-89). Barcelona.

Tardif, M. (2004) Los saberes del docente y su desarrollo profesional. Madrid: Narcea.

Zeichner, K. (1998a) Para além da divisão entre professor-pesquisador e pesquisador acadêmico En C. Gerladi, D. Fiorentini y E. Pereira (Eds.), Cartografia do trabalho docente: professor(a)-pesquisador(a) (pp. 207236). Campinas: Mercado de Letras. 
164 LA INVESTIGACIÓN SOBRE FORMACIÓN DOCENTE EN CHILE. TERRITORIOS EXPLORADOS E INEXPLORADOS - Tatiana Cisternas

Zeichner, K. (1998b) Tendências da pesquisa sobre formação de professores nos Estados Unidos. Revista Brasileira de Educação, 9, 76-87.

Zeichner, K. (1999) The New Scholarship in Teacher Education. Educational Researcher, 28(9), 4-15.

Recibido: 30/09/2011

Aceptado: 11/11/2011 